\title{
Update on Appropriate Use Criteria for Amyloid PET Imaging: Dementia Experts, Mild Cognitive Impairment, and Education
}

\author{
Keith A. Johnson ${ }^{1}$, Satoshi Minoshima ${ }^{2}$, Nicolaas I. Bohnen ${ }^{3}$, Kevin J. Donohoe ${ }^{4}$, Norman L. Foster ${ }^{5}$, Peter Herscovitch ${ }^{6}$, \\ Jason H. Karlawish ${ }^{7}$, Christopher C. Rowe ${ }^{8}$, Saima Hedrick ${ }^{9}$, Virginia Pappas ${ }^{9}$, Maria C. Carrillo ${ }^{10}$, and Dean M. Hartley ${ }^{10}$ \\ ${ }^{I}$ Departments of Radiology and Neurology, Massachusetts General Hospital, Harvard Medical School, Boston, Massachusetts; \\ ${ }^{2}$ Department of Radiology, University of Washington, Seattle, Washington; ${ }^{3}$ Departments of Radiology and Neurology, University of \\ Michigan, and VA Ann Arbor Healthcare System, Ann Arbor, Michigan; ${ }^{4}$ Beth Israel Deaconess Medical Center, Boston, \\ Massachusetts; ${ }^{5}$ Department of Neurology, University of Utah, Salt Lake City, Utah; ${ }^{6}$ PET Department, NIH Clinical Center, National \\ Institutes of Health, Bethesda, Maryland; ${ }^{7}$ Department of Medicine, University of Pennsylvania, Philadelphia, Pennsylvania; \\ ${ }^{8}$ Department of Nuclear Medicine and Centre for PET, Austin Health, Victoria, Australia; ${ }^{9}$ Society of Nuclear Medicine and \\ Molecular Imaging, Reston, Virginia; and ${ }^{10}$ Division of Medical and Scientific Relations, Alzheimer's Association, Chicago, Illinois
}

Amyloid PET imaging is a novel diagnostic test that can detect in living humans one of the two defining pathologic lesions of Alzheimer disease, amyloid- $\beta$ deposition in the brain. The Amyloid Imaging Task Force of the Alzheimer's Association and Society for Nuclear Medicine and Molecular Imaging previously published appropriate use criteria for amyloid PET as an important tool for increasing the certainty of a diagnosis of Alzheimer disease in specific patient populations. Here, the task force further clarifies and expands 3 topics discussed in the original paper: first, defining dementia experts and their use of proper documentation to demonstrate the medical necessity of an amyloid PET scan; second, identifying a specific subset of individuals with mild cognitive impairment for whom an amyloid PET scan is appropriate; and finally, developing educational programs to increase awareness of the amyloid PET appropriate use criteria and providing instructions on how this test should be used in the clinical decision-making process.

Key Words: amyloid PET; dementia; Alzheimer disease

J Nucl Med 2013; 54:1011-1013

DOI: 10.2967/jnumed.113.127068

A Alzheim living humans one of the defining pathologic lesions of Alzheimer disease, amyloid- $\beta$ deposition in the brain. We, the Amyloid Imaging Task Force of the Alzheimer's Association and Society for Nuclear Medicine and Molecular Imaging, convened to gather and evaluate published evidence and identify the circumstances under which amyloid PET would have important

Received May 31, 2013; accepted May 31, 2013.

For correspondence or reprints contact: Maria C. Carrillo, Division of Medical and Scientific Relations, Alzheimer's Association, 225 N. Michigan Ave., Chicago, IL 60601.

E-mail: maria.carrillo@alz.org

Published online Jun. 10, 2013.

COPYRIGHT @ 2013 by the Society of Nuclear Medicine and Molecular Imaging, Inc., and the Alzheimer's Association.

This article is being published jointly in The Journal of Nuclear Medicine and Alzheimer's \& Dementia. clinical utility. After nearly a year of evidence review and discussion, we concluded that the appropriate use of amyloid PET is limited to a subset of cognitively impaired patients, and then only under specific circumstances. Our appropriate use criteria (AUC) are described and supported in detail in a published report (1) but state in essence that amyloid PET is appropriately used only by a dementia expert as a single piece of information to support or oppose a clinical diagnosis of Alzheimer disease dementia or predementia in a patient in whom cognitive impairment has been objectively verified, in whom there is substantial uncertainty as to the underlying pathology, and for whom greater diagnostic certainty would change management.

We wish to supplement our recommendations on 3 specific issues as a result of discussions that have taken place since our report was published.

\section{DEMENTIA EXPERTS AND DOCUMENTATION}

\section{Dementia Experts}

We considered the role of the dementia expert to be critical and decisive to appropriately integrating imaging results with other clinical information. We carefully considered how to practically identify physicians with the requisite expertise. We concluded that clinical training and substantial clinical experience and practice in dementia care should be required. This level of expertise and familiarity is needed because amyloid PET is not a test for dementia or clinical Alzheimer disease but rather is an indicator of underlying pathology, which can be useful only when a particular clinical syndrome has been identified and other explanations have been adequately considered after a proper clinical evaluation. A high level of clinical experience with elderly patients and cognitive impairment is needed for the dementia expert to understand and properly apply the multifaceted consensus clinical criteria that define specific phases of Alzheimer disease dementia and predementia (1-4).

In response to particular interest expressed about our report since it appeared (1), we have reconvened and considered the specifications for a dementia expert in greater detail. There is no universally accepted definition of a dementia expert. Nevertheless, we concluded that a dementia expert for this purpose should be 
self-identified as a physician trained and board-certified in neurology, psychiatry, or geriatric medicine who devotes a substantial proportion $(\geq 25 \%)$ of patient contact time to the evaluation and care of adults with acquired cognitive impairment or dementia, including probable or suspected Alzheimer disease, as confirmed by peer recognition. Physicians particularly likely to have these characteristics are those with fellowship training or board certification in the emerging fields of geriatric psychiatry, geriatric neurology, and behavioral neurology and psychiatry. Although at present these subspecialists are few, the growing elderly population requires a pool of experts with proficiency in the assessment of cognitive impairment and with expertise to properly and selectively integrate imaging technology into clinical decision making. Our definition of a dementia expert is likely to include physicians who can recognize the appropriate subset of patients, refer them to a qualified PET facility, and then apply and communicate the information obtained according to the AUC. The current level of expertise among these clinicians will be enhanced through ongoing educational programs that the Alzheimer's Association, the Society of Nuclear Medicine and Molecular Imaging (SNMMI), and other professional organizations have already implemented.

We recognized, on the one hand, that because of the current shortage of dementia experts, the overall availability of amyloid PET will likely be narrow. On the other hand, the AUC indicate that amyloid PET is relevant only in a subset of cognitively impaired individuals in whom diagnosis remains uncertain after a complete evaluation. This is the subset of patients who are already likely to be referred to a dementia expert. Most initial work-up and management of suspected dementia is now in the hands of primary care providers and will undoubtedly remain there. However, this activity represents only a small fraction of a typical primary provider's practice; consequently, these providers often lack the experience or motivation to develop expertise in evaluating atypical and uncertain cases relevant to amyloid PET. For the appropriate use of amyloid PET, the expertise of the provider ordering the study and using the results to guide patient care is as critical as the technical quality and interpretation of the PET data. Dementia experts recognize that amyloid PET data must be carefully integrated with clinical data from detailed cognitive assessment and knowledgeable informant reporting. To the extent that proficiency with these integrative skills is not available in primary care practices, the inappropriate use of amyloid PET will be more likely to occur.

We concluded that the number of dementia experts would increase to serve the demand for amyloid PET referral if indeed amyloid PET can enable more effective management. We expect that interest in developing the requisite expertise will occur naturally as the technology is used in practice over time. Thus, we concluded that any initial constraint on amyloid PET due to the limited availability of the dementia expert will gradually resolve and that requiring a dementia expert to order the scan is justified now to maximize the potential that it will be used only under appropriate circumstances. After the medical community acquires greater experience in using amyloid PET, and training and expertise in cognitive evaluations increase, these limitations might be reconsidered.

\section{Documentation for Amyloid PET}

We developed a checklist to ensure appropriate use of amyloid PET, to standardize referral by dementia experts, and to ensure that patients considered for this study have undergone a standard clinical evaluation and treatment of comorbidities before the scan is ordered. The dementia expert should collect and share with the PET physician the following information, which should be maintained in the patient medical record for extraction and review:

Age and date of onset of symptoms;

$\square$ Identification of clinical syndrome (mild cognitive impairment, or MCI; dementia);

$\square$ Clinical evidence of persistent or progressive cognitive decline;

$\square$ Structured objective assessment of mental status such as the Mini-Mental State Examination, Montreal Cognitive Assessment, or a similar measure;

$\square$ Known comorbidities;

$\square$ List of prescribed medications and rationale for use of psychoactive medications, if any;

$\square$ Results of neuropsychologic testing performed;

$\square$ Results of structural brain imaging (such as MR imaging or CT) performed;

$\square$ Relevant laboratory tests, which should include complete blood count, chemistry profile, B12, and thyroid hormone;

$\square$ Reasons that the cause of cognitive impairment remains uncertain after completion of a standard clinical evaluation and treatment of comorbidities;

$\square$ Treatment and care plan based on amyloid PET findings.

We recommend that the dementia expert and PET physician be required to document this information in the medical record of each patient. Third-party payers should consider this information fully sufficient to demonstrate the medical necessity of amyloid PET, and this information could be submitted to payers on request. This information also could be the basis for sponsorship of a voluntary registry for independent assessment of appropriate use and for targeting of educational efforts.

\section{$\mathrm{MCl}$}

The recent SNMMI/Alzheimer's Association AUC emphasize that amyloid PET for patients with persistent or progressive unexplained MCI may be appropriate under limited and specific circumstances. These guidelines emphasize that "not all patients with MCI would be appropriate for amyloid PET. Rather, amyloid PET would be appropriate only in those $[\mathrm{MCI}]$ individuals who the dementia expert has concluded would benefit from greater certainty of the underlying pathology and whose clinical management would change as a result of this greater certainty" (1). The above checklist will be applied to this patient group for the purpose of limiting referrals for amyloid PET to appropriate patients. Amyloid PET is indicated only for those who, according to the dementia expert, carry a diagnosis of MCI that conforms to established consensus criteria as discussed above (indication 1 in the AUC document (1-4)). We considered that the scope of use of amyloid PET would be substantially limited by 2 prerequisites imposed by the AUC, the requirement for etiologic uncertainty and the requirement for a change in patient management.

\section{Etiologic Uncertainty}

Sources of etiologic uncertainty - which may arise in any patient being evaluated for MCI or Alzheimer disease dementia-are well known to the dementia expert. They are typically clinical issues that can be evaluated with established methods and usually do not ultimately interfere with proper diagnosis and management. 
For example, uncertainty may arise when the clinical onset of the syndrome is atypical or sudden, when the illness may be etiologically mixed because of vascular pathology or evidence of possible Lewy pathology, or when potentially confounding circumstances are involved, such as depression, substance abuse, medication effects, and cardiovascular, endocrine, or other medical disorders. Occasionally, however, the dementia expert is confronted by complexities such as these and cannot determine the likelihood that amyloid- $\beta$ pathology is ultimately responsible for the impairment. In these relatively unusual but important cases, substantial uncertainty then remains as to the cause of the syndrome, and further patient care is severely constrained or compromised. The treating physician must then adopt a wait-and-see approach that is unsatisfying, potentially misguided, at times wasteful, and potentially harmful.

\section{Change in Patient Management}

Because disease-modifying treatments for Alzheimer disease have not yet been established, the dementia expert is stymied by a limited range of management alternatives. Nevertheless, important choices are made by the treating physician, the patient, and caregivers on the basis of the presence or absence of detectable amyloid- $\beta$. Once the cause of deficits is properly assessed, a more confident diagnosis frequently helps anticipate future needs, including closer monitoring for progression and safety, periodic functional assessments for retirement planning, and allocation of financial and family resources. For management decisions such as these, we concluded that amyloid PET could be appropriately used "when knowledge of the presence or absence of [amyloid- $\beta$ ] pathology is expected to increase diagnostic certainty and alter management" (1).

\section{EDUCATION}

We are committed to assisting professional organizations in creating educational materials to ensure appropriate use of amyloid PET, in increasing awareness of the criteria among physicians and patients, and in providing instructions on how to incorporate amyloid PET findings into clinical decision making. To ensure appropriate use of amyloid PET, several educational activities are currently ongoing through the Alzheimer's Association and the SNMMI.

\section{National Meetings}

The SNMMI has established a session devoted to amyloid PET and related technology at its annual mid-winter meetings. In addition, the SNMMI added several sessions, including a "Read with the Expert" session for amyloid PET, to its 2013 annual meeting in Vancouver and will be doing the same at subsequent annual meetings.

\section{Local Meetings}

The SNMMI continues to organize numerous regional meetings to provide information on amyloid PET for both physicians and technologists.

\section{Educational Materials}

The SNMMI is developing educational materials for Maintenance of Certificate modules for physicians. The SNMMI in collaboration with the European Association of Nuclear Medicine is developing a procedure guideline to ensure high-quality imaging techniques for amyloid PET. The Alzheimer's Association is developing educational materials that will be shared with the organization's national distribution of chapters for guiding patients, caregivers, and physicians on seeking a dementia specialist and the appropriate uses of amyloid PET.

\section{Educational Outreach}

The Outreach Committee at the SNMMI has organized sessions at professional societies advocating appropriate use of amyloid PET. Such a session will be included at the 2013 annual meetings of the American Academy of Neurology and the American Association of Geriatric Psychiatry. Similar sessions are planned for the annual meetings of the American Academy of Family Physicians and the American Psychiatric Association. An online education program is being developed to include didactic materials for neurologists, geriatricians, and psychiatrists. The Alzheimer's Association has developed talking points that are used in nationwide presentations to patients, caregivers, and physicians to educate these individuals on the appropriate uses of amyloid PET. These outreach efforts will continue to ensure appropriate use of amyloid PET for the patients who most benefit from this imaging procedure.

\section{DISCLOSURE}

No potential conflict of interest relevant to this article was reported.

\section{REFERENCES}

1. Johnson KA, Minoshima S, Bohnen NI, et al. Appropriate use criteria for amyloid PET: a report of the Amyloid Imaging Task Force, the Society of Nuclear Medicine and Molecular Imaging, and the Alzheimer's Association. Dual publication in: Alzheimers Dement. 2013;9:e-1-16 and J Nucl Med. 2013;54:476-490.

2. McKhann GM, Knopman DS, Chertkow H, et al. The diagnosis of dementia due to Alzheimer's disease: recommendations from the National Institute on AgingAlzheimer's Association workgroups on diagnostic guidelines for Alzheimer's disease. Alzheimers Dement. 2011;7:263-269.

3. Albert MS, DeKosky ST, Dickson D, et al. The diagnosis of mild cognitive impairment due to Alzheimer's disease: recommendations from the National Institute on Aging-Alzheimer's Association workgroups on diagnostic guidelines for Alzheimer's disease. Alzheimers Dement. 2011;7:270-279.

4. Dubois B, Feldman HH, Jacova C, et al. Revising the definition of Alzheimer's disease: a new lexicon. Lancet Neurol. 2010;9:1118-1127. 